\title{
Electromagnetic Waves Dispersion and Interaction of an Annular Beam-Ion Channel System in Plasma Waveguide
}

\author{
Jixiong Xiao, ${ }^{1,2}$ Zhijiang Wang, ${ }^{1}$ Jun Chen, ${ }^{2}$ and Changhai Liu ${ }^{1}$ \\ ${ }^{1}$ Institute of Fusion and Plasma Research, Huazhong University of Science and Technology, Luoyu Road 1037, Hubei, \\ Wuhan 430074, China \\ ${ }^{2}$ School of Electrical and Electronic Engineering, Hubei University of Technology, Nan Li Road 28, Hubei, Wuhan 430068, China \\ Correspondence should be addressed to Jixiong Xiao; xiaojixiong0738@163.com
}

Received 30 June 2017; Revised 13 October 2017; Accepted 31 October 2017; Published 20 November 2017

Academic Editor: Prabir Daripa

Copyright (C) 2017 Jixiong Xiao et al. This is an open access article distributed under the Creative Commons Attribution License, which permits unrestricted use, distribution, and reproduction in any medium, provided the original work is properly cited.

\begin{abstract}
A linear theory for the electromagnetic properties and interactions of an annular beam-ion channel system in plasma waveguide is presented. The dispersion relations for two families of propagating modes, including the electrostatic and transverse magnetic modes, are derived. The dependencies of the dispersion behavior and interaction for different wave modes on the thickness of the annular beam and betatron oscillation frequency are studied in detail by numerical calculations. The results show that the inner and outer radii of the beam have different influences on propagation properties of the electrostatic and electromagnetic modes with different betatron oscillation parameters. In the weak ion channel situation, the two types of electrostatic waves, that is, space charge and betatron modes, have no interaction with the transverse magnetic modes. However, in the strong ion channel situation, the transverse magnetic modes will have two branches and a low frequency mode emerged as the new branch. In this case, compared with the solid beam case, the betatron modes not only can interact with the high frequency branch at small wavenumber but also can interact with the low frequency branch at large wavenumber.
\end{abstract}

\section{Introduction}

As is well known, plasma filling has a variety of advantages in increasing the space charge limited current, overall energy conversion efficiency, and radiation bandwidth dramatically [1-3] and it has been widely used in many plasma microwave radiation sources, such as traveling-wave tubes (TWT) [4], backward-wave oscillators (BWO) [5], klystrons [6], and gyrotrons [7]. Compared with the vacuum electronic devices, the EM dispersion characteristic and beam-wave energy transfer mechanism will become more complex [8]. As it is the basis of the application of any plasma electronics devices, the EM dispersion behavior and interaction in such plasma apparatus have always been research hot spots in the past two decades. In the past years, most of the published papers concentrated on the case of the relativistic electron beam (REB) with an external magnetic field guiding. In recent years, ion channel guiding has been put forward as an innovative focusing method for guiding the electron bunch transport $[9$, 10]. To form an ion channel, the electron beam density $n_{b}$ and the plasma density $n_{p}$ must satisfy the matching condition $n_{b} / \gamma^{2} \leq n_{p}<n_{b}[9,11]$. Here, $\gamma$ is Lorentz factor of the beam. As a REB passes through the preformed plasma, the beam front will push out the plasma electrons continuously by the space charge force induced by beam electron, leaving the almost immobile positive ions to form the so-called ion channel. Then the electron beam can transport reliably with the guidance of the focus force of the ion channel. This new focusing method has been experimentally demonstrated in plasma wave tubes (PWT) [12], free electron lasers (FEL) [13], and charged particle accelerators [14] successfully. In previous works, the high frequency azimuthally symmetrical and nonsymmetrical eigenmodes in a REB with ion-channel guiding have been analyzed by Rouhani et al. $[15,16]$. Wang et al. have studied the dispersion relations of EM waves in beam-ion channel system and the mechanism of Cherenkov EM instability [17, 18]. Mirzanejhad et al. have presented the dispersion characteristics of the space charge waves in a uniform and rigid rotation REB in ion channel without betatron oscillations [19]. 


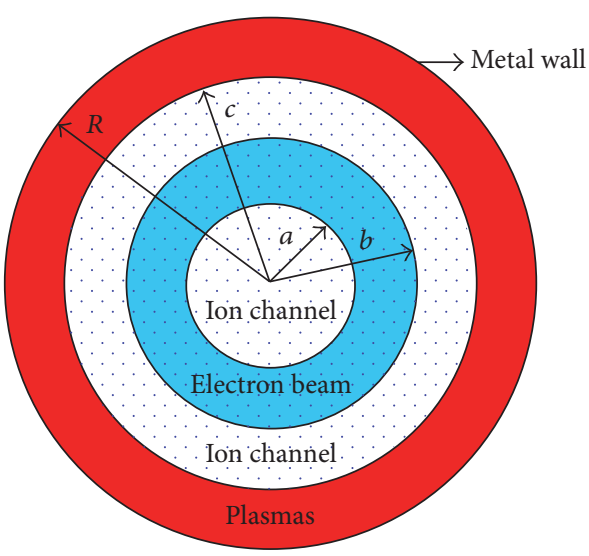

FIGURE 1: The cross section of the annular beam-ion channel system. The annular electron beam with inner radius $a$ and outer radius $b$, ion channel with radius $c$, and metallic waveguide with radius $R$.

However, these papers mainly focused on the solid beam case. As annular beam has higher space charge limiting current and beam-wave energy conversion efficiency, it is also used widely. Therefore, the aim of the present article is the investigation of the EM wave dispersion and interaction of an annular beam-ion channel system in cylindrical plasma waveguide. There are three types of eigenmodes that will be discussed and the electrostatic (ES) and EM approximation will be used to study the space charge modes, betatron modes, and transverse magnetic (TM) modes, respectively. After dispersion relations are derived, the influences of the beam bunch thickness and betatron oscillation parameter on the dispersion propagation property and interaction of different wave modes are discussed in detail by numerical calculations. The differences between the solid and annular beam are also given by a comparison.

The remainder of the article is structured as follows. In Section 2, the governing equations and the solutions of the wave equations in different regions for ES and TM waves are derived in the beam coordinate system and the dispersion relations are also presented. In Section 3, a numerical study of azimuthally symmetric eigenmodes is presented. In Section 4 , some conclusions are made.

\section{Basic Equations and Dispersion Relations}

A cylindrical metallic waveguide with radius $R$ is completely filled with an annular plasma column. This plasma column is with inner radius $a$ and outer radius $R$. A relativistic annular electron beam with inner radius $a$ and outer radius $b$ passes through it with an initial velocity $v_{b 0 z}$ along with the waveguide axis. The annular beam expels the plasma electron continuously and forms an ion channel with radius $c$ in the background plasma. The schematic diagram has been shown in Figure 1.

In the ion channel, the beam is subjected to three forces in the radial direction, which are originated from the actions of the transverse electric field $\mathbf{E}_{i}$ produced by the positive ion core, the space charge field $\mathbf{E}_{b}^{s}$ of the REB,

$$
\begin{aligned}
\alpha & =\left[\frac{\omega_{b}^{2}}{\left(\omega^{2}-\gamma \omega_{i}^{2} / 2\right)}\right]-1, \\
A(x, y) & =J_{0}(x) N_{0}(y)-J_{0}(y) N_{0}(x), \\
B(x, y) & =J_{0}(x) N_{1}(y)-J_{1}(y) N_{0}(x), \\
C(x, y) & =J_{1}(x) N_{1}(y)-J_{1}(y) N_{1}(x), \\
D(x, y) & =I_{0}(x) K_{0}(y)-I_{0}(y) K_{0}(x), \\
E(x, y) & =I_{0}(x) K_{1}(y)+I_{1}(y) K_{0}(x),
\end{aligned}
$$

and the azimuthal self-magnetic field $\mathbf{B}_{b}^{s}$ induced by the beam current. Applying the Gauss' theorem and Ampere's theorem, $\mathbf{E}_{i}, \mathbf{E}_{b}^{s}$, and $\mathbf{B}_{b}^{s}$ can be expressed as follows, respectively:

$$
\begin{aligned}
\mathbf{E}_{i} & =\frac{e n_{i} r}{2 \varepsilon_{0}} \mathbf{e}_{r}, \\
\mathbf{E}_{b}^{s} & =-\frac{e n_{b}}{2 \varepsilon_{0}} \frac{r^{2}-a^{2}}{r} \mathbf{e}_{r}, \\
\mathbf{B}_{b}^{s} & =-\frac{e v_{b 0 z} n_{b}}{2 c^{2} \varepsilon_{0}} \frac{r^{2}-a^{2}}{r} \mathbf{e}_{\theta} .
\end{aligned}
$$

Here, $n_{i}$ is the ion density and is equal to the plasma density $n_{p}, n_{b}$ is the electron beam density, $e$ is the unit charge, $r$ is the radial coordinate, $\mathbf{e}_{r}$ is the radial unit vector, and $\mathbf{e}_{\theta}$ is the azimuthal unit vector. The radial force balance equation is

$$
\gamma m_{e} \frac{d r^{2}}{d t^{2}}=-e\left[\frac{e n_{i}}{2 \varepsilon_{0}} r-\left(\frac{e n_{b}}{2 \varepsilon_{0}}-\frac{e n_{b} v_{b 0 z}^{2}}{2 \varepsilon_{0} c^{2}}\right) \frac{r^{2}-a^{2}}{r}\right] .
$$

If the inner radius $a$ approaches zero, the annular beam will reduce to the solid beam case and the solution has been given in [15], which can be written as $\mathbf{r}=\mathbf{r}_{0} \cos \left(\omega_{i} t / \sqrt{2 \gamma}\right)$, and $\mathbf{r}_{0}$ denotes the initial radial coordinate. The equation of motion of the electron beam is

$$
\frac{d \mathbf{p}}{d t}=-e(\mathbf{E}+\mathbf{v} \times \mathbf{B})
$$

Note that $d / d t=\partial / \partial t+(\mathbf{v} \cdot \nabla)$. Here, we only discuss linear waves; it means that the amplitude of oscillation of the waves is small and the terms containing higher powers of amplitude factors can be neglected. Therefore, we can assume that all physical quantities can be expressed as $\mathbf{f}=\mathbf{f}_{0}+\mathbf{f}_{1} \exp [j(\omega t-$ $\left.\left.k_{z} z+l \theta\right)\right], \mathbf{f}_{0}$ and $\mathbf{f}_{1}$ represent the equilibrium quantity and perturbation quantity, respectively, and $\mathbf{f}_{1} \ll \mathbf{f}_{0}$. Therefore, we have $\mathbf{r}=\mathbf{r}_{b 0}+\mathbf{r}_{1}, \mathbf{v}=\mathbf{v}_{b 0 z}+\mathbf{v}_{b 1}, \mathbf{E}=\mathbf{E}_{i}+\mathbf{E}_{b}^{s}+\mathbf{E}_{1}$, and $\mathbf{B}=\mathbf{B}_{b}^{s}+\mathbf{B}_{1}$. If introducing cylindrical coordinates $(r, \theta, z)$ and $z$-axis coincides with the waveguide axes, the transverse 
and longitudinal motion equation of the beam electrons can be, respectively, expressed as

$$
\begin{aligned}
& {\left[\frac{\partial}{\partial t}+\left(\mathbf{v}_{b 0 z}+\mathbf{v}_{b 1}\right) \cdot \nabla\right] \mathbf{v}_{b 1 \perp}=-\frac{e}{\gamma m_{e}}\left\{\mathbf{E}_{1 \perp}+\frac{n_{p} e}{2 \varepsilon_{0}}\left(\mathbf{r}_{b 0}\right.\right.} \\
& \left.+\mathbf{r}_{b 1}\right)-\frac{n_{b} e}{2 \varepsilon_{0}}\left(\mathbf{r}_{b 0}+\mathbf{r}_{b 1}-\frac{a^{2} \mathbf{e}_{r}}{r_{b 0}+r_{b 1}}\right)+\left[\left(\mathbf{v}_{b 0 z}+\mathbf{v}_{b 1}\right)\right. \\
& \left.\quad \times \mathbf{B}_{1}\right]_{\perp}-\frac{n_{b} e v_{b 0 z}}{2 \varepsilon_{0} c^{2}}\left[\left(\mathbf{v}_{b 0 z}+\mathbf{v}_{b 1}\right)\right. \\
& \left.\left.\quad \times\left(\mathbf{r}_{b 0}+\mathbf{r}_{b 1}-\frac{a^{2} \mathbf{e}_{r}}{r_{b 0}+r_{b 1}}\right)\right]_{\perp}\right\}, \\
& \left.\frac{\partial}{\partial t}+\left(\mathbf{v}_{b 0 z}+\mathbf{v}_{b 1}\right) \cdot \nabla\right] \mathbf{v}_{b 1 z}=-\frac{e}{\gamma m_{e}}\left\{\mathbf{E}_{1 z}\right. \\
& \quad+\left[\left(\mathbf{v}_{b 0 z}+\mathbf{v}_{b 1}\right) \times \mathbf{B}_{1}\right]_{z}-\frac{n_{b} e v_{b 0 z}}{2 \varepsilon_{0} c^{2}}\left[\left(\mathbf{v}_{b 0 z}+\mathbf{v}_{b 1}\right)\right. \\
& \left.\left.\quad \times\left(\mathbf{r}_{b 0}+\mathbf{r}_{b 1}-\frac{a^{2}}{r_{b 0}+r_{b 1}}\right)\right]_{z}\right\}+e \frac{v_{b 0 z}^{2}}{\gamma m_{e} c^{2}} \mathbf{E}_{1 z} \cdot
\end{aligned}
$$

In the above derivation we have considered the relativistic effects and must notice that

$$
\begin{aligned}
\frac{d \mathbf{p}}{d t} & =\gamma m_{e}\left(\frac{\partial \mathbf{v}_{b 1}}{\partial t}+\mathbf{v}_{b 1} \cdot \nabla \mathbf{v}_{b 1}\right)+m_{e} v_{b 0 z} \frac{\partial \gamma}{\partial t} \\
m_{e} v_{b 0 z} \frac{\partial \gamma}{\partial t} & =\frac{v_{b 0 z}}{c^{2}} \frac{d W}{d t} .
\end{aligned}
$$

Here, $W=\gamma m c^{2}$ is the relativistic energy. Using the relation $d W / d t=\mathbf{F} \cdot \mathbf{V}$ results in

$$
m_{e} v_{b 0 z} \frac{\partial \gamma}{\partial t}=-e \frac{v_{b o z}^{2}}{c^{2}} \mathbf{E}_{1 z} .
$$

In (5), $\mathbf{r}_{b 0}$ is used to describe the betatron oscillation in the radial direction and $\mathbf{r}_{b 1}$ is used to describe the radial displacement induced by high frequency fields. The subscripts " $\perp$ " and " $z$ " denote the transverse and longitudinal component, respectively. If without disturbances all the beam electrons are nearly on the $z$-axis at the initial time, the perturbation amplitude caused by betatron oscillation will be nearly identical with the perturbation induced by high frequency fields so that both of them can be considered as a perturbation. Based on this assumption, after linearization, we can obtain the first-order motion equation:

$$
\begin{aligned}
\left(\frac{\partial}{\partial t}+\mathbf{v}_{b 0 z} \frac{\partial}{\partial z}\right) \mathbf{v}_{b 1}= & -\frac{e}{\gamma m_{e}}\left(\mathbf{E}_{1 \perp}+\mathbf{v}_{b 0 z} \times \mathbf{B}_{1}\right)-\frac{\omega_{i}^{2}}{2 \gamma} \mathbf{r}_{1} \\
& -\frac{e \mathbf{E}_{1 z}}{\gamma^{3} m_{e}} .
\end{aligned}
$$

In the comoving coordinate of the beam, although the background plasma will be equivalent to a moving plasma column, the processing of the moving electron beam can be avoided, which is beneficial to simplify the derivation. Transformation to the beam frame (8) can be written as

$$
\frac{\partial}{\partial t} \mathbf{v}_{b 1}=-\frac{e}{m_{e}} \mathbf{E}_{1}-\frac{\gamma \omega_{i}^{2}}{2} \mathbf{r}_{1} .
$$

\subsection{Electrostatic Modes. The Poisson equation is}

$$
\nabla^{2} \widetilde{\varphi}=\frac{e n_{b 1}}{\varepsilon_{0}} .
$$

The linearized continuity equation is

$$
\frac{\partial}{\partial t} n_{b 1}+n_{b 0}^{b} \nabla \cdot \mathbf{v}_{b 1}=0
$$

where $n_{b 0}^{b}=n_{b} / \gamma$ is the unperturbed density of the REB in the beam frame.

Noticing that $\mathbf{v}_{\perp}=d \mathbf{r} / d t=-j \omega \mathbf{r}$, the transverse component of (9) can be written as

$$
\left(\frac{\partial}{\partial t}-\frac{\gamma \omega_{i}^{2}}{2 j \omega}\right) v_{b 1 \perp}=-\frac{e}{m_{e}} \nabla_{\perp} \tilde{\varphi} .
$$

By using (10), (11), and (12), the ES waves equation in the beam region can be obtained as

$$
\nabla_{\perp}^{2} \tilde{\varphi}+T^{2} \tilde{\varphi}=0
$$

where

$$
T^{2}=\frac{k_{z}^{2}\left(\omega^{2}-\gamma \omega_{i}^{2} / 2\right)\left(\omega^{2}-\omega_{b}^{2}\right)}{\omega^{2}\left(-\omega^{2}+\omega_{b}^{2}+\gamma \omega_{i}^{2} / 2\right)}
$$

and $\omega_{b}$ is the beam frequency in beam frame. For the vacuum and ion channel regions, we can get

$$
\nabla_{\perp}^{2} \tilde{\varphi}-k_{z}^{2} \widetilde{\varphi}=0
$$

Therefore, the suitable solutions in the different regions in the waveguide can be expressed as, respectively,

$$
\widetilde{\varphi}= \begin{cases}c_{1} I_{l}\left(k_{z} r\right) & 0<r \leq a \\ c_{2} J_{l}(T r)+c_{3} N_{l}(T r) & a<r \leq b \\ c_{4} I_{l}\left(k_{z} r\right)+c_{5} K_{l}\left(k_{z} r\right) & b<r \leq R,\end{cases}
$$

where $J_{l}$ is the Bessel function of the first kind, $N_{l}$ is the Bessel function of the second kind, and $I_{l}$ and $K_{l}$ are modified Bessel functions. Applying the appropriate boundary conditions [20], we can get a set of equations for five unknown coefficients. Eliminating the unknown coefficients, the ES wave dispersion relation can be obtained as

$$
\begin{aligned}
& \frac{I_{1}\left(k_{z} a\right)}{I_{0}\left(k_{z} a\right)}=\alpha \frac{T}{k_{z}} \\
& \quad \cdot \frac{k_{z} E\left(k_{z} R, k_{z} b\right) B(T b, T a)+T \alpha D\left(k_{z} R, k_{z} b\right) C(T b, T a)}{k_{z} E\left(k_{z} R, k_{z} b\right) A(T b, T a)-T \alpha D\left(k_{z} R, k_{z} b\right) B(T a, T b)} .
\end{aligned}
$$


Here, only symmetric modes $(l=0)$ are considered and, in the above equation, we have introduced

$$
\begin{aligned}
\alpha & =\left[\frac{\omega_{b}^{2}}{\left(\omega^{2}-\gamma \omega_{i}^{2} / 2\right)}\right]-1, \\
A(x, y) & =J_{0}(x) N_{0}(y)-J_{0}(y) N_{0}(x), \\
B(x, y) & =J_{0}(x) N_{1}(y)-J_{1}(y) N_{0}(x), \\
C(x, y) & =J_{1}(x) N_{1}(y)-J_{1}(y) N_{1}(x), \\
D(x, y) & =I_{0}(x) K_{0}(y)-I_{0}(y) K_{0}(x), \\
E(x, y) & =I_{0}(x) K_{1}(y)+I_{1}(y) K_{0}(x) .
\end{aligned}
$$

Equation (17) is the dispersion relation for space charge and betatron waves with ES approximation. If the annular beam fills the waveguide completely, the dispersion relation will reduce to

$$
\frac{I_{1}\left(k_{z} a\right)}{I_{0}\left(k_{z} a\right)}=\frac{T \alpha}{k_{z}} \frac{B(T R, T a)}{A(T R, T a)}
$$

If the inner radius of the annular beam is close to zero, the annular beam will reduce to the solid beam case

$$
\frac{J_{1}(T b)}{J_{0}(T b)}=\frac{k_{z}}{T \alpha} \frac{E\left(k_{z} R, k_{z} b\right)}{D\left(k_{z} R, k_{z} b\right)} .
$$

2.2. TM Modes. After linearization, Maxwell's equations are

$$
\begin{aligned}
\nabla \times \mathbf{E}_{1} & =j \omega \mathbf{B}_{1}, \\
\nabla \times \mathbf{B}_{1} & =-\mu_{0} e n_{b 0}^{b} \mathbf{v}_{b 1}-j \omega \mu_{0} \varepsilon_{0} \mathbf{E}_{1}, \\
\nabla \cdot \mathbf{E}_{1} & =-\frac{e n_{b 1}}{\varepsilon_{0}}, \\
\nabla \cdot \mathbf{B}_{1} & =0 .
\end{aligned}
$$

By using (21) and (22), we can find the transverse and longitudinal equation for $\mathbf{E}_{1}$ as follows:

$$
\begin{aligned}
& -\frac{\partial}{\partial z}\left(\frac{\partial E_{1 r}}{\partial z}-\frac{\partial E_{1 z}}{\partial r}\right)-\frac{\omega^{2}}{c^{2}} E_{1 r}=-j \omega \mu_{0} e n_{b 0}^{b} v_{b 1 r} \\
& \frac{1}{r} \frac{\partial}{\partial r}\left(r\left(\frac{\partial E_{1 r}}{\partial z}-\frac{\partial E_{1 z}}{\partial r}\right)\right)-\frac{\omega^{2}}{c^{2}} E_{1 z} \\
& =-j \omega \mu_{0} e n_{b 0}^{b} v_{b 1 z}
\end{aligned}
$$

From (9) and (25), the TM wave equation in the beam region can be obtained as

$$
\begin{aligned}
& \nabla_{\perp}^{2} E_{1 z}+T^{2} E_{1 z}=0, \\
& T^{2} \\
& =\frac{\left(\omega^{2}-\omega_{b}^{2}\right)\left[k_{z}^{2}\left(\gamma \omega_{i}^{2} / 2-\omega^{2}\right)-\left(\omega^{2} / c^{2}\right)\left(\omega_{b}^{2}-\omega^{2}+\gamma \omega_{i}^{2} / 2\right)\right]}{\omega^{2}\left(\omega^{2}-\omega_{b}^{2}-\gamma \omega_{i}^{2} / 2\right)} .
\end{aligned}
$$

In the beam coordinate system, the quasi-static background plasma can be viewed as a moving medium with a velocity $v_{b 0 z}$ along the $z$-axis negative direction. Using linear theory, we can get the expressions of the transverse and longitudinal perturbation current after a simple derivation, respectively,

$$
\begin{gathered}
\mathbf{J}_{p 1 \perp}=-j \omega \varepsilon_{0}\left\{-\frac{\omega_{p}^{2}}{\omega^{2}} \mathbf{E}_{p 1 \perp}-j \frac{\omega_{p}^{2}}{\left(\omega+k_{z} v_{b 0 z}\right)} \frac{v_{b 0 z}}{\omega^{2}}\right. \\
\left.\cdot \nabla_{\perp} E_{p 1 z}\right\}, \\
\mathbf{J}_{p 1 z}=-j \omega \varepsilon_{0}\left\{\left(\frac{-\omega_{p}^{2}}{\left(\omega+k_{z} v_{b 0 z}\right) \gamma^{2}}\right.\right. \\
\left.+\frac{\omega_{p}^{2} v_{b 0 z}^{2}}{\left(\omega+k_{z} v_{b 0 z}\right)^{2} \omega^{2}} \nabla_{\perp}^{2}\right) \mathbf{E}_{p 1 z}-j \\
\left.\cdot \frac{\omega_{p}^{2} \mathbf{v}_{b 0 z}}{\left(\omega+k_{z} v_{b 0 z}\right) \omega^{2}}\left(\nabla_{\perp} \cdot \mathbf{E}_{p 1 \perp}\right)\right\} .
\end{gathered}
$$

Ampere's circuital law is

$$
\nabla \times \mathbf{B}_{1}=\mu_{0} \mathbf{J}_{1}-j \omega \varepsilon_{0} \mu_{0} \mathbf{E}_{1}=-j \omega \mu_{0} \overrightarrow{\vec{\varepsilon}} \cdot \mathbf{E}_{1} .
$$

Therefore, the equivalent dielectric tensor of the plasma column can be obtained as

$$
\overrightarrow{\boldsymbol{\varepsilon}}=\varepsilon_{0}\left(\begin{array}{ccc}
\varepsilon_{1} & 0 & j \varepsilon_{2} \nabla_{r} \\
0 & \varepsilon_{1} & j \varepsilon_{2} \nabla_{\theta} \\
j \varepsilon_{2} \nabla_{r} & j \varepsilon_{2} \nabla_{\theta} & \varepsilon_{3}
\end{array}\right),
$$

where $\varepsilon_{1}=1-\omega_{p}^{2} / \omega^{2}, \varepsilon_{2}=-\omega_{p}^{2} v_{b 0 z} /\left(\omega+k_{z} v_{b 0 z}\right) \omega^{2}$, and $\varepsilon_{3}=1-\omega_{p}^{2} /\left(\omega+k_{z} v_{b 0 z}\right)^{2} \gamma^{2}+\left(\omega_{p}^{2} v_{b o z}^{2} /\left(\omega+k_{z} v_{b 0 z}\right)^{2} \omega^{2}\right) \nabla_{\perp}^{2}$.

And the wave equation in the plasma region can be obtained as

$$
\nabla_{\perp}^{2} E_{1 z}+p^{2} E_{1 z}=0
$$

here, $p^{2}=\left(\omega^{2}-\omega_{p}^{2}\right) / c^{2}-k_{z}^{2}$.

For high frequency EM waves, the ion channel region can be regarded as vacuum and the wave equation in this region is

$$
\nabla_{\perp}^{2} E_{1 z}+\tau^{2} E_{1 z}=0
$$

and $\tau^{2}=\omega^{2} / c^{2}-k_{z}^{2}$.

The appropriate solutions for (26), (31), and (32) in the different regions of the waveguide are of the form

$$
\begin{array}{ll}
E_{1 z}=c_{1} J_{l}(\tau r) \quad 0<r \leq a, & \\
E_{2 z}=c_{2} J_{l}(T r)+c_{2} N_{l}(T r) & a<r \leq b, \\
E_{3 z}=c_{4} J_{l}(\tau r)+c_{5} N_{l}(\tau r) & b<r \leq c, \\
E_{4 z}=c_{6} J_{l}(p r)+c_{7} N_{l}(p r) & c<r \leq R .
\end{array}
$$


Applying the appropriate boundary conditions [20], the TM modes dispersion equation can be obtained as follows:

$$
\frac{J_{0}^{\prime}(\tau a)}{J_{0}(\tau a)}=A_{0} \frac{A_{1} B(p R, p c) X_{1}+A(p R, p c) X_{2}}{A(p R, p c) X_{3}-A_{1} B(p R, p c) X_{4}}
$$

Here, only symmetric modes $(l=0)$ are considered. In the above equation, we have introduced

$$
\begin{aligned}
& A_{0}=\frac{\tau}{T} \frac{\omega^{2}-\omega_{b}^{2}}{\omega^{2}}, \\
& A_{1}=\left[\varepsilon_{1}-\varepsilon_{2} k_{z}\right] \frac{\tau}{p},
\end{aligned}
$$

$$
\begin{aligned}
& X_{1}=A_{0} C(T b, T a) A(\tau b, \tau c)+B(T b, T a) B(\tau c, \tau b), \\
& X_{2}=B(T b, T a) C(\tau b, \tau c)-A_{0} C(T b, T a) B(\tau b, \tau c), \\
& X_{3}=A_{0} B(T a, T b) B(\tau b, \tau c)-A(T a, T b) C(\tau b, \tau c), \\
& X_{4}=A(T a, T b) B(\tau c, \tau b)+A_{0} B(T a, T b) A(\tau b, \tau c) .
\end{aligned}
$$

In laboratory coordinate system the expression of the ion channel radius is of the form $r=\Delta R\left(n_{b} / n_{p}\right)^{1 / 2}[21]$ and $\Delta R$ is the thickness of the beam. It will increase with the thickness of the beam $\Delta R$ or the reciprocal of the plasma neutralization factor $f=n_{p} / n_{b}$. If it fills the waveguide completely, in this case, the dispersion relation will be rewritten as

$$
\frac{J_{0}^{\prime}(\tau a)}{J_{0}(\tau a)}=\frac{\tau}{T} \frac{\omega^{2}-\omega_{b}^{2}}{\omega^{2}} \frac{(\tau / T)\left(\left(\omega^{2}-\omega_{b}^{2}\right) / \omega^{2}\right) C(T b, T a) A(\tau R, \tau b)-B(\tau R, \tau b) B(T b, T a)}{B(\tau R, \tau b) A(T a, T b)-(\tau / T)\left(\left(\omega^{2}-\omega_{b}^{2}\right) / \omega^{2}\right) B(T a, T b) A(\tau R, \tau b)} .
$$

When the waveguide filled with the annular beam completely, (36) will reduce to

$$
\frac{J_{0}^{\prime}(\tau a)}{J_{0}(\tau a)}=\frac{\tau}{T} \frac{\omega^{2}-\omega_{b}^{2}}{\omega^{2}} \frac{B(T R, T a)}{A(T R, T a)} .
$$

If the inner radius of the annular beam is close to zero, the annular beam will reduce to the solid beam case

$$
\begin{aligned}
& \frac{J_{0}^{\prime}(T b)}{J_{0}(T b)}=\frac{1}{A_{0}} \\
& \quad \cdot \frac{A_{1} B(p R, p c) B(\tau c, \tau b)+A(p R, p c) C(\tau b, \tau c)}{B(\tau b, \tau c) A(p R, p c)-A_{1} B(p R, p c) A(\tau b, \tau c)} .
\end{aligned}
$$

\section{Numerical Results}

In this part, a numerical calculation is used to analyze the effects of the inner and outer radii on the dispersion curves of the azimuthally symmetric $(l=0)$ ES and TM modes and the interaction between them under different EM characteristics of the ion channel. For the convenience of the numerical calculation, The dispersion frequency $\omega$ and the wavenumber $k_{z}$ are normalized by the beam frequency $\omega_{b}$ and the speed of the light in vacuum; that is, $\widetilde{\omega}=\omega / \omega_{b}$ and $\widetilde{k}_{z}=k_{z} c / \omega_{b}$. The normalized waveguide radius has the value $R \omega_{b} / c=1.2$, which corresponds to $R=2 \mathrm{~mm}$ and $\omega_{b}=1.8 \times 10^{11} \mathrm{rad} / \mathrm{s}$ with the beam density $n_{b 0}^{b}=1.033 \times 10^{13} \mathrm{~cm}^{-3}$ in the beam coordinate system and these values are consistent with [15].

From (14) and (27), we can find that the transverse oscillation characteristic frequency $\omega_{\beta}=\sqrt{\gamma / 2} \omega_{i}$ has important influence on the solution of the ES and TM mode dispersion equation. For example, $\omega_{\beta}$ is larger or smaller than the beam frequency $\omega_{b}$; the dispersion equations of the ES and TM modes will have different solutions and it leads to the wave modes in both cases having different dispersion characteristics. Based on this point it can be classified as strong and weak ion channel and in the cases of the weak and strong ion channel, which meet $\sqrt{1 / \gamma}<\omega_{p} / \omega_{b} \leq \sqrt{3 / \gamma}$ and $\sqrt{3 / \gamma}<\omega_{p} / \omega_{b} \leq \sqrt{\gamma}$, respectively.

The asymptotic behaviors and the influences of the inner and outer radii on the space charge and betatron modes for strong ion channel are presented in Figures 2-5. In this case the dispersion curves of the $\mathrm{SC}_{01}$ waves will shift to the beam frequency $\omega_{b}$ as the wavenumber is close to infinity and the $\mathrm{Be}_{01}$ waves have upper hybrid frequency $\omega_{H}=\left(\omega_{b}^{2}+\gamma \omega_{i}^{2} / 2\right)^{1 / 2}$ [15]. It can also be found that, as the inner radius decreased or the outer radius increased, the $\mathrm{SC}_{01}$ modes experience obviously shifts close to higher frequencies but the case of the $\mathrm{Be}_{01}$ modes is just to the opposite and its dispersion curves shift down clearly. Besides, compared with the solid beam case, the $\mathrm{SC}_{01}$ modes have higher frequencies and the $\mathrm{Be}_{01}$ modes have lower frequencies in the annular beam case.

Figures 6-9 have shown the asymptotic behaviors and the effects of the inner and outer radii on space charge and betatron modes for weak ion channel. In this case $\mathrm{SC}_{01}$ waves have upper hybrid frequency $\omega_{H}=\left(\omega_{b}^{2}+\gamma \omega_{i}^{2} / 2\right)^{1 / 2}$ and will approach the beam frequency $\omega_{b}$ with the wavenumber being close to infinity, and $\mathrm{Be}_{01}$ waves have the cutoff frequency $\omega_{\beta}$. We can also see that from the figures, in contrast to the strong channel case, the roles of the space charge and betatron modes will be exchanged in weak ion channel so that the effects of the inner and outer radii on the space charge and betatron modes are also exchanged.

Figures 2-9 also show that, in both of the strong and weak ion channel case, the asymptotic behaviors of the $\mathrm{SC}_{01}$ and $\mathrm{Be}_{01}$ modes for the case of the solid beam in [15] coincide with the annular beam case in this paper. Besides, the beam radius of the solid beam and the outer radius of the annular beam have a similar effect on the space and betatron modes. As the solid beam can be regarded as a special case of the inner radius of the annular beam close to zero, we can theoretically expect such results. 


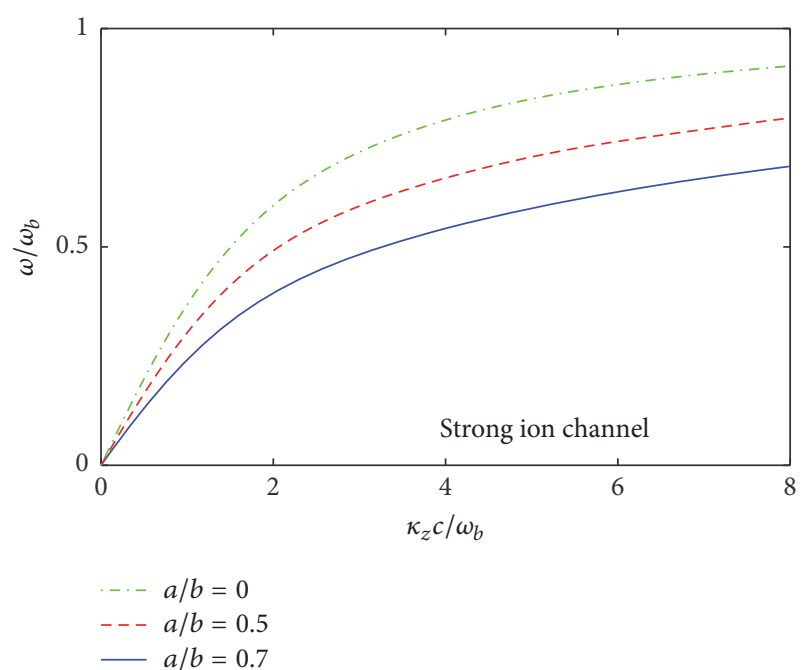

FIgURE 2: The influence of the ratio $a / b$ on the dispersion curves of the $\mathrm{SC}_{01}$ waves for strong ion channel with $\omega_{\beta}=2.3195$. The outer radius of the annular beam is taken as $0.5 R$ and $a / b=0$ represents the solid beam case.

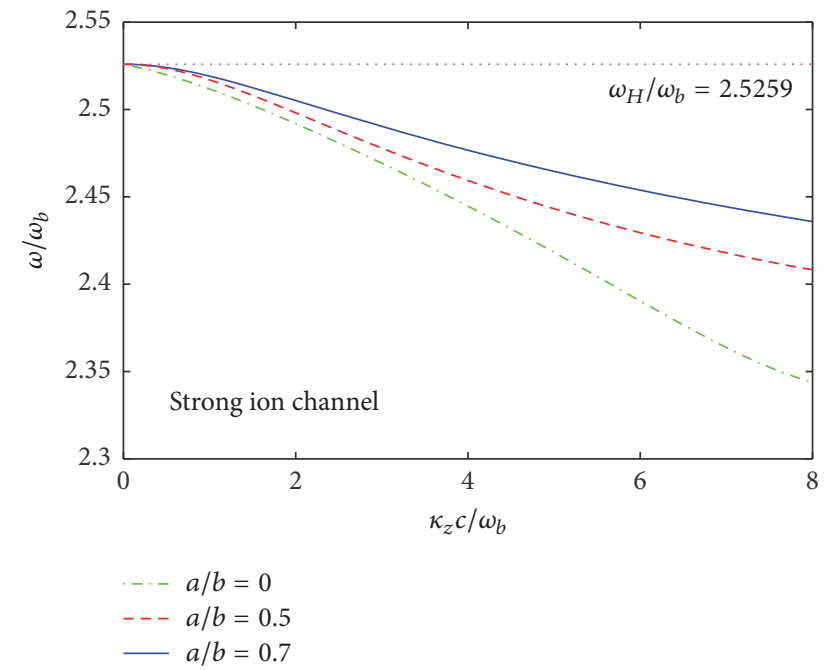

FIgURE 3: The influence of the ratio $a / b$ on the dispersion curves of the $\mathrm{Be}_{01}$ waves for strong ion channel with $\omega_{\beta}=2.3195$. The outer radius of the annular beam is taken as $0.5 R$ and $a / b=0$ represents the solid beam case.

The influences of the inner radius on the $\mathrm{TM}_{01}$ waves in the cases of strong and weak ion channel are demonstrated in Figures 10 and 11. With the increasing of the inner radius (the ratio $a / b$ ), the dispersion curves of the $\mathrm{TM}_{01}$ modes will undergo slightly frequency shifts toward higher frequencies for both of the strong and weak ion channel and the upward shifts are more obscurely in strong ion channel case. In general, the EM eigenmode in an annular beam system has a higher phase velocity than the solid beam case with the same condition [22]. Therefore, the TM modes have higher frequencies for the solid case.

The TM modes normalized dispersion frequencies as a function of the ratio of the outer radius $b$ to the waveguide

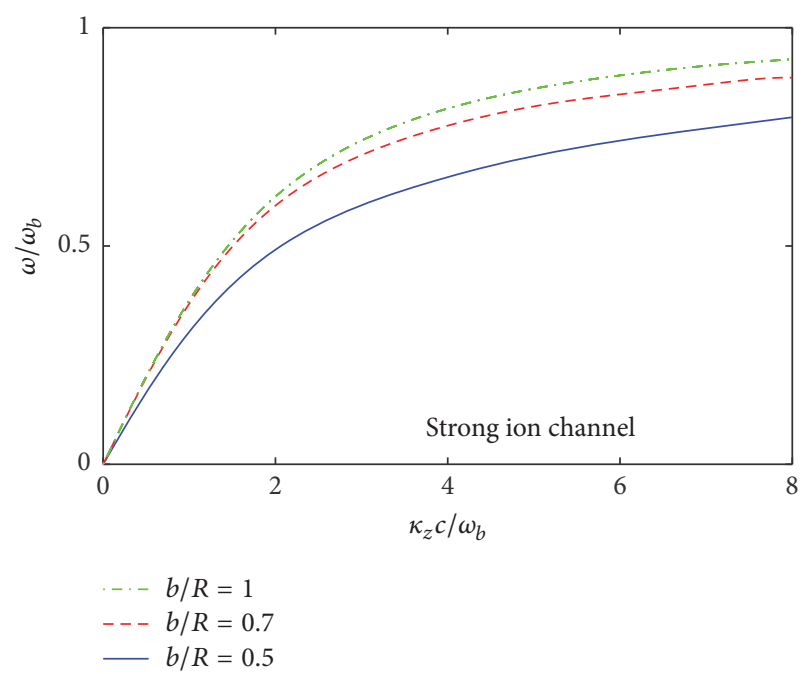

FIGURE 4: The influence of the ratio $b / R$ on the dispersion curves of the $\mathrm{SC}_{01}$ waves for strong ion channel with $\omega_{\beta}=2.3195$. The inner radius of the annular beam is taken as $0.25 R . b / R=1$ represents the waveguide completely filled with the annular beam.

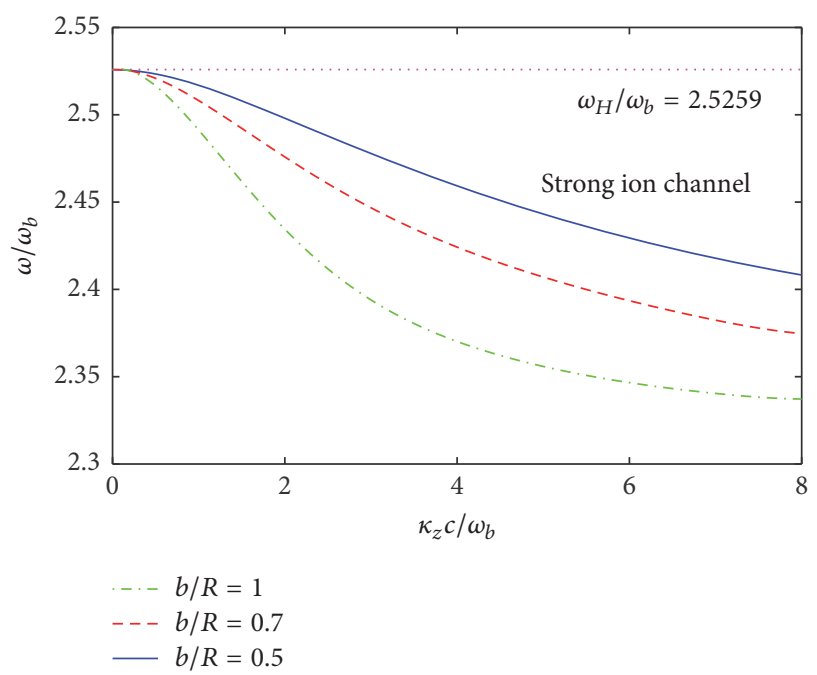

FIGURE 5: The influence of the ratio $b / R$ on the dispersion curves of the $\mathrm{Be}_{01}$ waves for strong ion channel with $\omega_{\beta}=2.3195$. The inner radius of the annular beam is taken as $0.25 R . b / R=1$ represents the waveguide completely filled with the annular beam.

radius $R$ are illustrated in Figure 12. In this figure, $\omega_{\beta} / \omega_{b}=$ 1.6205 and $\omega_{\beta} / \omega_{b}=0.8832$ correspond to $\omega_{i} / \omega_{b}=1.1456$ and $\omega_{i} / \omega_{b}=0.6245$, respectively. As the radius of the ion channel is proportional to the thickness, that is, $r=$ $\Delta R\left(\sqrt{\gamma} \omega_{b} / \omega_{p}\right)$ in beam frame, the ion channel will fill the waveguide gradually with the increasing of the out radius. In this case, we can find that if $b / R>0.46$ or $b / R>0.6625$, the radius of the ion channel will be larger than the waveguide radius $R$, which means that it will fill the waveguide completely. As this figure shows, while the waveguide is only filled with it partially, the TM modes frequencies will shift down gradually as the outer radius increased. However, if the 


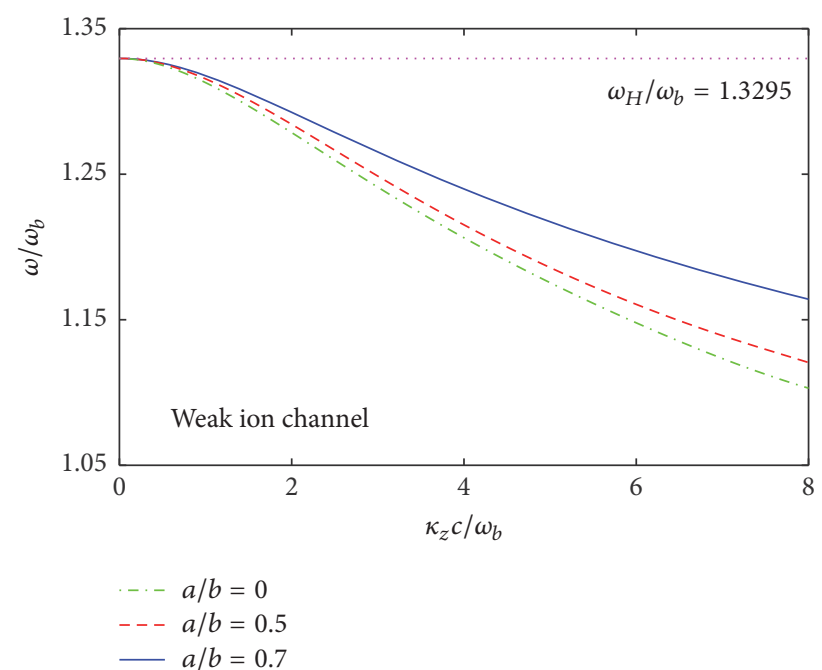

FIgURE 6: The effect of the ratio $a / b$ on the dispersion curves of the $\mathrm{SC}_{01}$ waves for weak ion channel with $\omega_{\beta}=0.8761$. The outer radius of the annular beam is taken as $0.5 R$ and $a / b=0$ represents the solid beam case.

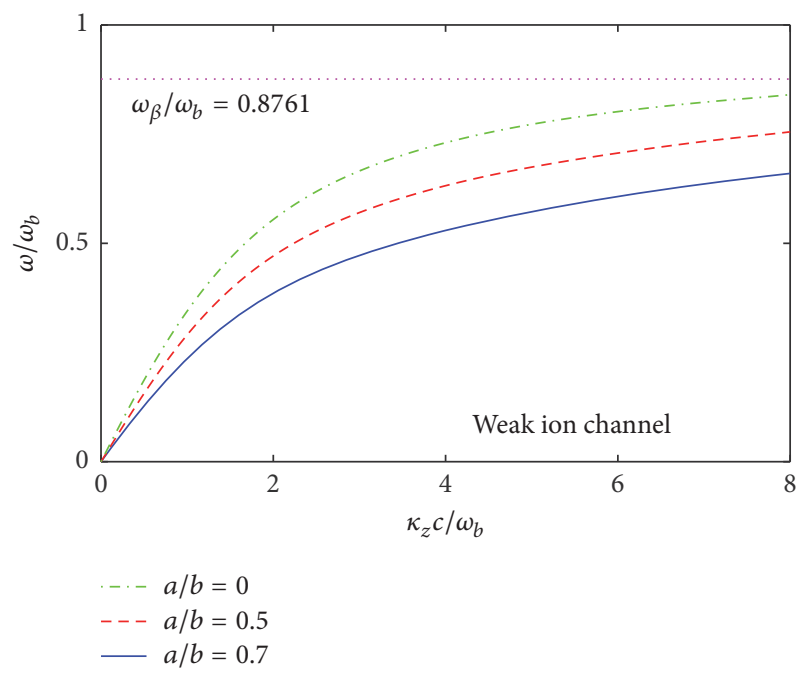

FIGURE 7: The effect of the ratio $a / b$ on the dispersion curves of the $\mathrm{Be}_{01}$ waves for weak ion channel with $\omega_{\beta}=0.8761$. The outer radius of the annular beam is taken as $0.5 R$ and $a / b=0$ represents the solid beam case.

waveguide is filled with it completely, this case is just to the opposite of the partially filled case and the frequencies will shift up.

Figure 13 shows the influences of the betatron oscillation characteristic parameter $\omega_{\beta}$ on the TM dispersion properties for strong ion channel. With the increasing of $\omega_{\beta}$, there will be two branches for TM modes and a low frequency (LF) mode with a lower cutoff frequency emerged as the new branch. We can also find that the dispersion behavior of this new branch is similar to the high frequency branch at small wavenumber, but it is similar to betatron modes at large wavenumber. It is supposed that the reason of this phenomenon is that the dispersion equation (34) will have a new solution with the

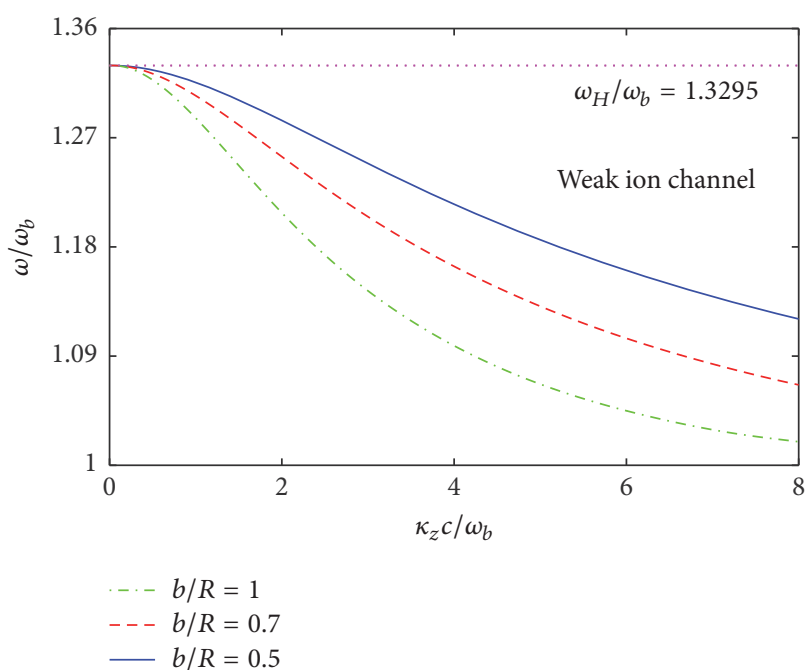

FIgURE 8: The effect of the ratio $b / R$ on the dispersion curves of the $\mathrm{SC}_{01}$ modes for weak ion channel with $\omega_{\beta}=0.8761$. The inner radius of the annular beam is taken as $0.25 R . b / R=1$ represents the waveguide completely filled with the annular beam.

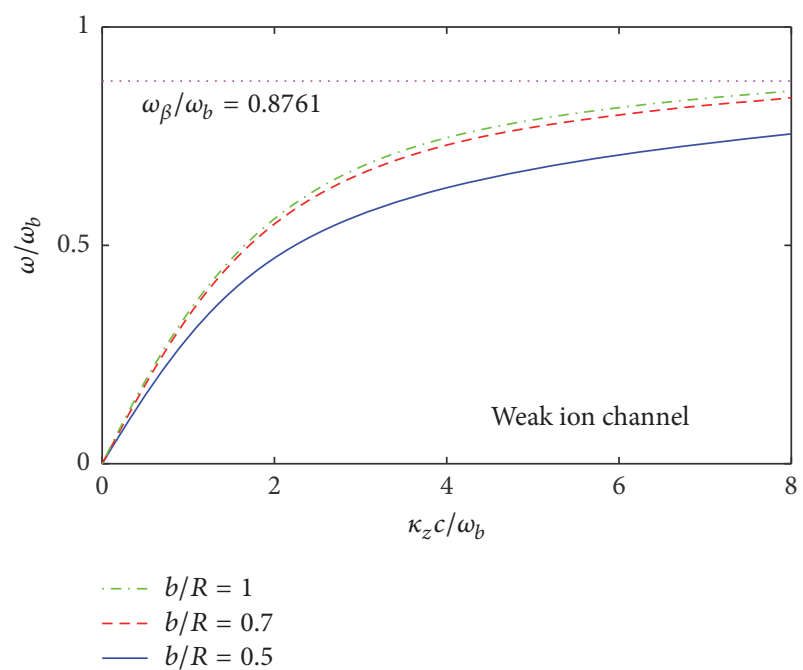

FIgURE 9: The effect of the ratio $b / R$ on the dispersion curves of the $\mathrm{Be}_{01}$ modes for weak ion channel with $\omega_{\beta}=0.8761$. The inner radius of the annular beam is taken as $0.25 R . b / R=1$ represents the waveguide completely filled with the annular beam.

increase of the betatron oscillation frequency. Besides, as the longitudinal wavenumber $k_{z}$ approaches infinity, the new LF branch will have longitudinal wave-like character, which may result in the new LF branch having betatron-like character. This is very different from the solid beam case and we did not find similar phenomenon in that case.

The interactions between the TM and ES modes for the cases of the annular beam and solid are presented in Figures 14-17. As can be seen from the figures, the cutoff frequencies for the $\mathrm{SC}_{01}$ and $\mathrm{Be}_{01}$ waves are lower than the TM wave in the weak ion channel case so that there is no coupling between the three types propagating modes. However, the 


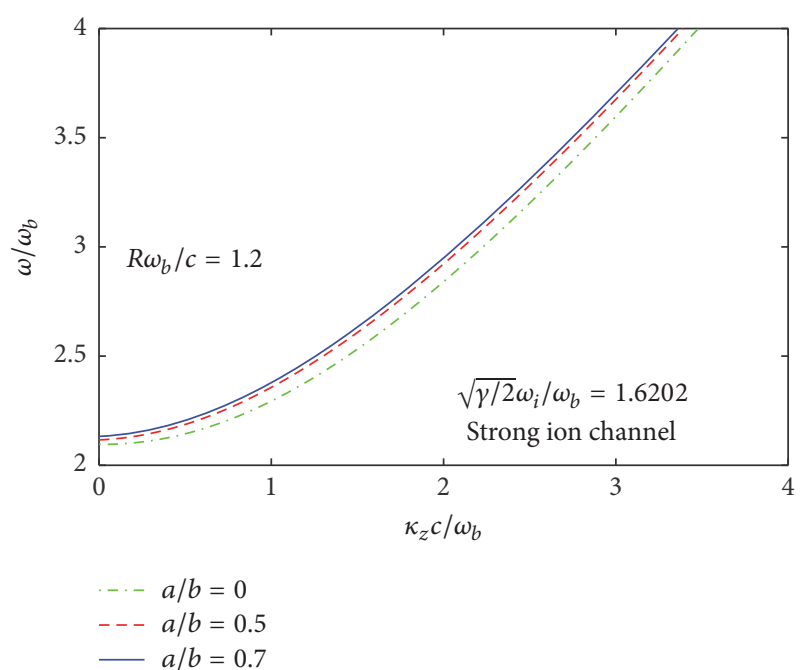

FIGURE 10: The effect of the ratio $a / b$ on the dispersion curves of the $\mathrm{TM}_{01}$ modes for strong ion channel with $\omega_{\beta}=1.6202$. The outer radius of the annular beam is taken as $b=0.4 R$ and $a / b=0$ represents the solid beam case.

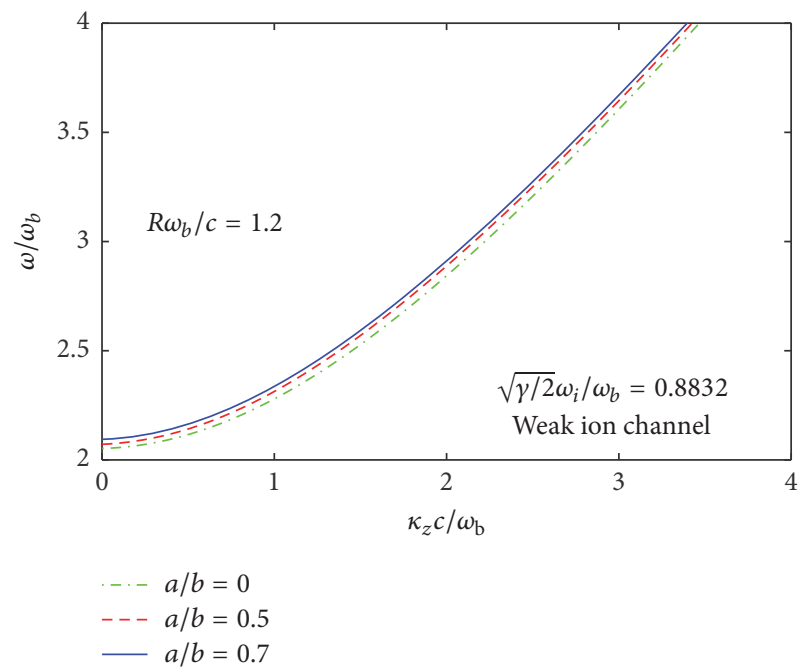

FIGURE 11: The effect of the ratio $a / b$ on the dispersion curves of the $\mathrm{TM}_{01}$ modes for weak ion channel with $\omega_{\beta}=0.8832$. The outer radius of the annular beam is taken as $b=0.4 R$ and $a / b=0$ represents the solid beam case.

strong ion channel case is completely different. Although the asymptotic frequency $\omega_{b}$ of the $\mathrm{SC}_{01}$ modes is far smaller than the cutoff frequency of the TM modes and it cannot interact with the TM modes, the $\mathrm{Be}_{01}$ modes can interact with the TM modes for both the annular and solid beam cases. Compared with the solid beam, from Figure 15, it can also be seen that the dispersion curve of the LF branch for the TM modes is close to the betatron modes gradually and the two dispersion curves almost coincident at large number wave. This is an interesting phenomenon. As the betatron waves are backward waves and have negative group velocity, the coupling between

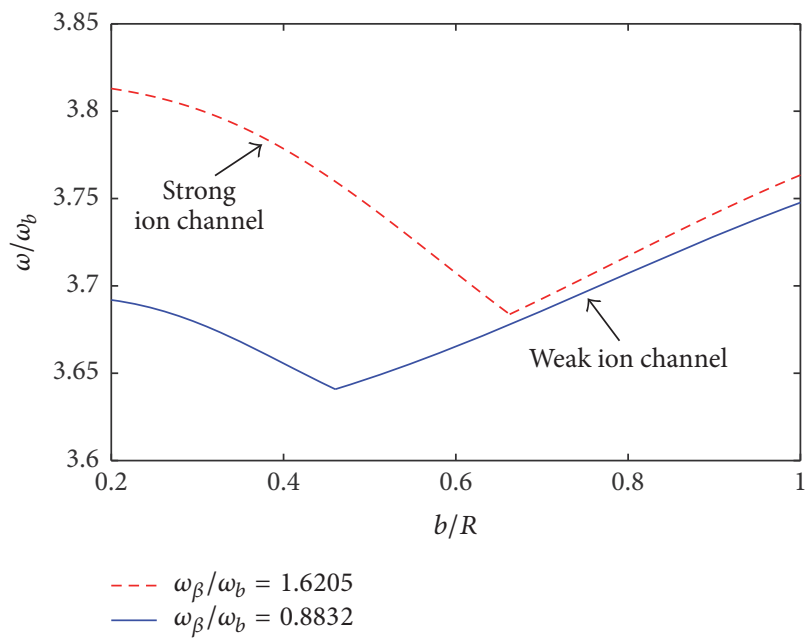

FIGURE 12: The effect of the ratio $b / R$ on the dispersion curves of the TM modes for strong and weak ion channel with $\gamma=4$. The inner radius of the annular beam is taken as $a=0.1 R$.

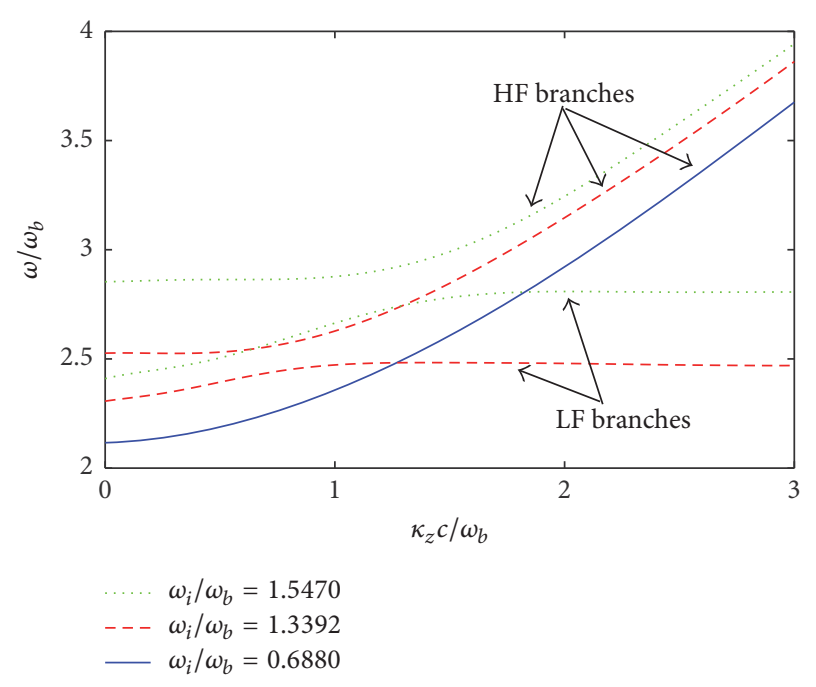

FIGURE 13: The effect of betatron oscillation parameter $\omega_{\beta}$ on the dispersion characteristics of the $\mathrm{TM}_{01}$ modes for strong ion channel. The inner and outer radius of the annular beam are taken as $a=$ $0.25 R$ and $b=0.5 R$. The Lorentz factor is $\gamma=6$.

betatron and TM wave may produce absolute instability and this interaction can be used for microwave generation [23].

\section{Conclusion}

In present analysis, the EM dispersion properties and interactions of an annular beam-ion channel system in plasma waveguide are studied by linear perturbation theory. The influences of the inner and outer radii of the beam and betatron oscillation parameter on the dispersion characteristics of space charge modes, betatron modes, and TM modes are revealed by numerical calculations. It is shown that the inner and outer radii have different influences on the three types of waves under different betatron characteristic frequencies and the TM modes will have two branches in 


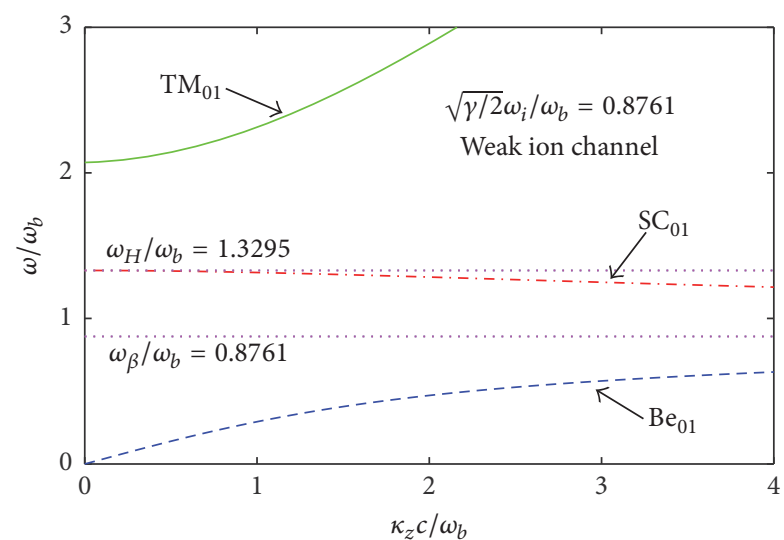

FIGURE 14: In annular beam case, there is no coupling between the TM modes and ES waves in weak ion channel case. The inner and outer radius of the annular beam are taken as $a=0.25 R$ and $b=$ $0.5 R$; the Lorentz factor is $\gamma=6$.

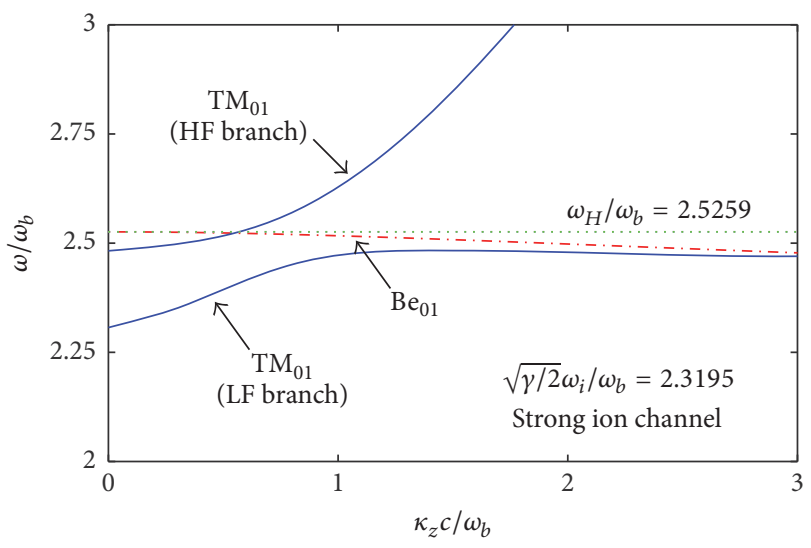

FIGURE 15: In annular beam case, the TM wave of the two branches can interact with the betatron wave in strong ion channel case. The inner and outer radius of the annular beam are taken as $a=0.25 R$ and $b=0.5 R$; the Lorentz factor is $\gamma=6$.

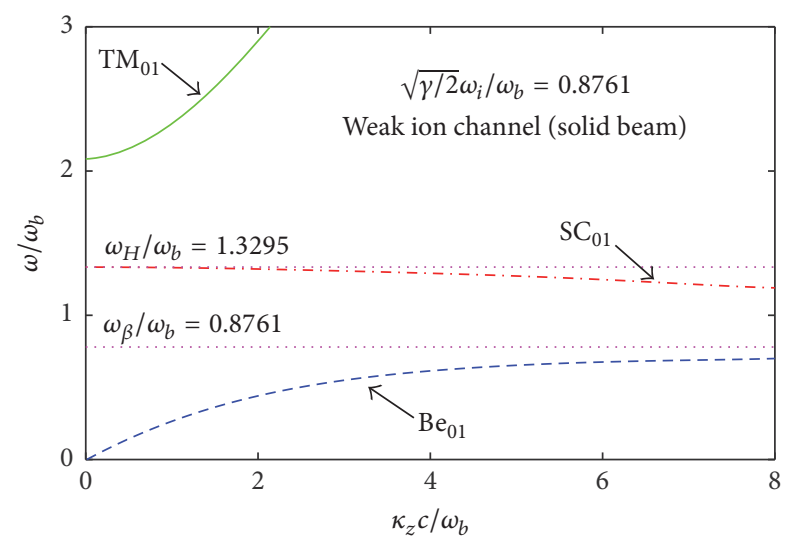

FIGURE 16: In solid beam case, there is no coupling between the TM and ES waves in weak ion channel case. The beam radius is taken as $a=0.25 R$; the Lorentz factor is $\gamma=6$.

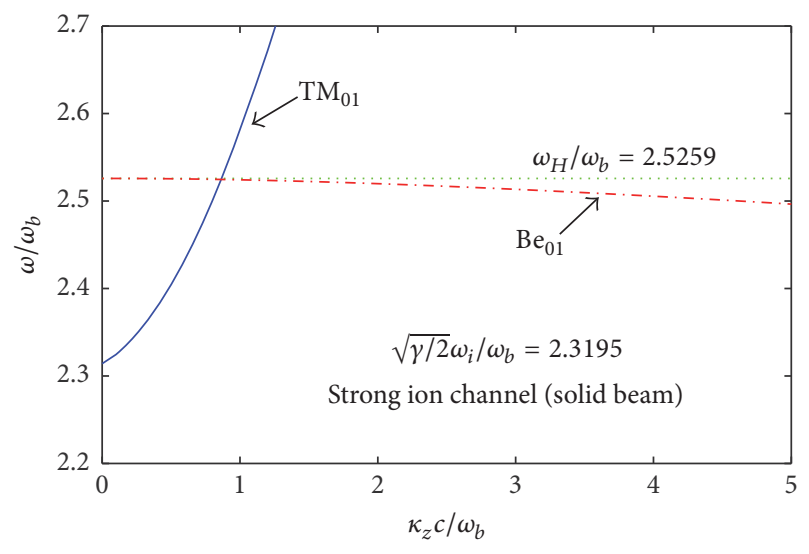

FIGURE 17: For solid beam, the TM wave only has one branch and can interact with the betatron wave in strong ion channel case. The beam radius is taken as $a=0.25 R$; the Lorentz factor is $\gamma=6$.

strong ion channel case with appropriate betatron frequency. The investigation of the interaction between the ES waves and TM waves for strong and weak ion channel indicates that there is no coupling between the two families propagating modes in weak ion channel case but the $\mathrm{Be}_{01}$ modes can interact with the both of the two branches of the $\mathrm{TM}_{01}$ waves in the strong ion channel case. The coupling between the low frequency branch and the betatron wave especially is a new phenomenon and the details still need further study. Obviously, it can be found from the conclusions of this paper that the ion channel on the one hand plays an important role of guiding the beam to ensure the transport effectively. On the other hand, different betatron oscillation characteristic frequency of the ion channel can make the propagating wave modes in the system show different EM characteristics and change the interaction between them. In addition, the physical model used in this paper also has some limitations; for example, the influence of the electron duration is not taken into account. On the one hand, for a short pulse electron beam, when the beam is injected into the plasma, a return current will be excited in it and the direction of the return current is opposite to the beam current so that it may have a significant impact on the balance of the electron beam in the radial direction and also can affect the eigenstructure of the system. On the other hand, for a long pulse electron, there may be oscillating charge on the boundary of the ion channel and then the oscillating current may be formed [24]. It may also affect the dispersion characteristics of the system. Therefore, these works still need further analyses in the future.

\section{Conflicts of Interest}

The authors declare that they have no conflicts of interest.

\section{Acknowledgments}

This work is supported by the National ITER Project Foundation of China (nos. 2013GB106001 and 2013GB106003). 


\section{References}

[1] D. M. Goebel, Y. Carmel, and G. S. Nusinovich, "Advances in plasma-filled microwave sources," Physics of Plasmas, vol. 6, no. 5 I, pp. 2225-2232, 1999.

[2] W. Fu and Y. Yan, "Harmonic generation of high-power microwave in plasma filled waveguide," International Journal of Infrared and Millimeter Waves, vol. 29, no. 1, pp. 43-50, 2008.

[3] X. Renzhen, L. Yuzheng, S. Zhimin, C. Changhua, and L. Guozhi, "Theoretical study of a plasma-filled relativistic Cerenkov generator with coaxial slow-wave structure," IEEE Transactions on Plasma Sciences, vol. 35, no. 5, pp. 1456-1466, 2007.

[4] S. Saviz, "Plasma thermal effect on the growth rate of the helix traveling wave tube," IEEE Transactions on Plasma Sciences, vol. 42, no. 8, pp. 2023-2029, 2014.

[5] L. Guo, T. Shu, Z. Li, H. Zhang, and J. Ju, "An efficient gigawatt level X -band Cerenkov type oscillator without guiding magnetic field," Physics of Plasmas, vol. 21, no. 7, Article ID 073106, 2014.

[6] J. Ju, J. Zhang, T. Shu, and H. Zhong, "An improved X-band triaxial klystron amplifier for gigawatt long-pulse high-power microwave generation," IEEE Electron Device Letters, vol. 38, no. 2, pp. 270-272, 2017.

[7] G. I. Zaginaylov, V. I. Shcherbinin, K. Schüenemann, and M. K. Thumm, "Influence of background plasma on electromagnetic properties of "Cold" gyrotron cavity," IEEE Transactions on Plasma Sciences, vol. 34, no. 3, pp. 512-517, 2006.

[8] F. Wenjie and Y. Yang, "Analysis of high-Power microwave propagation in a magnetized plasma filled waveguide," Plasma Science and Technology, vol. 15, no. 10, pp. 974-978, 2013.

[9] D. H. Whittum, W. M. Sharp, S. S. Yu, M. Lampe, and G. Joyce, "Electron-hose instability in the ion-focused regime," Physical Review Letters, vol. 67, no. 8, pp. 991-994, 1991.

[10] A. Taghavi, M. Esmaeilzadeh, and M. S. Fallah, "Chaotic electron trajectories in an electromagnetic wiggler free-electron laser with ion-channel guiding," Physics of Plasmas, vol. 17, no. 9, Article ID 093103, 2010.

[11] D. H. Whittum, "Electromagnetic instability of the ion-focused regime," Physics of Fluids, vol. 4, no. 3, pp. 730-739, 1992.

[12] J. Santoru, J. M. Butler, D. M. Goebel, and R. W. Schumacher, "Highpower, Single-Beam Plasma Wave Tube," IEEE Transactions on Plasma Sciences, vol. 22, no. 5, pp. 593-599, 1994.

[13] S. Sadegzadeh, A. Hasanbeigi, H. Mehdian, and M. Alimohamadi, "Linear theory of magnetized ion-channel freeelectron laser," Physics of Plasmas, vol. 19, no. 2, Article ID 023108, 2012.

[14] R. Bingham, J. T. Mendonça, and P. K. Shukla, "Plasma based charged-particle accelerators," Plasma Physics and Controlled Fusion, vol. 46, no. 1, pp. R1-R23, 2004.

[15] M. H. Rouhani and B. Maraghechi, "Wave-mode dispersions in a relativistic electron beam with ion-channel guiding," Physics of Plasmas, vol. 13, no. 8, Article ID 083101, 2006.

[16] B. Maraghechi and B. Maraghechi, "Dispersion and interaction of waveguide modes in a relativistic electron beam with ionchannel guiding," Physics of Plasmas, vol. 15, no. 7, Article ID 073112, 2008.

[17] Z.-Y. Wang, C.-J. Tang, and X.-D. Peng, "Cherenkov electromagnetic instability excited by an oscillating relativistic electron beam in ion channel," Physics of Plasmas, vol. 17, no. 8, Article ID 083114, 2010.
[18] H.-R. Li, C.-J. Tang, and S.-J. Wang, "Wave growth rate in a cylindrical metal waveguide with ion-channel guiding of a relativistic electron beam," Chinese Physics B, vol. 19, no. 12, Article ID 124101, 2010.

[19] S. Mirzanejhad, B. Maraghechi, F. Sohbatzadeh, and I. KamelJahromi, "Space-charge waves in a relativistic electron beam with ion-channel guiding," Journal of Plasma Physics, vol. 77, no. 3, pp. 419-429, 2011.

[20] J. D. Jackson, Classical Electrodynamics, Wiley Press, New York, NY, USA, 3rd edition, 1962.

[21] X. Wenkai, Theory and Design of Charged Particle Beams, Science Press, Beijing, China, 2009.

[22] Z. Rahmani, B. Jazi, and E. Heidari-Semiromi, "Terahertz electromagnetic wave generation and amplification by an electron beam in the elliptical plasma waveguides with dielectric rod," Physics of Plasmas, vol. 21, no. 9, Article ID 092122, 2014.

[23] L. Shenggang, Introduction to Microwave Electronics, National Defense industy Press, Beijing, China, 1985.

[24] J. Xiao, Z. Zeng, D. Xia, Z. Wang, and C. Liu, "Effects of boundary current on electromagnetic dispersion characteristics for a relativistic electron beam," Plasma Science and Technology, vol. 18, no. 1, pp. 51-57, 2016. 


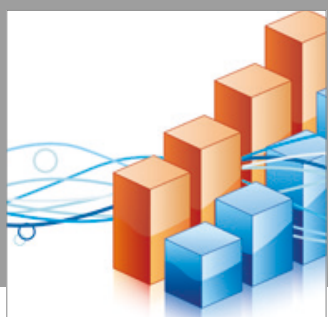

Advances in

Operations Research

vatersals

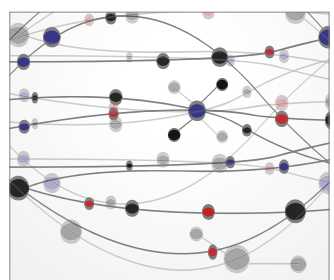

\section{The Scientific} World Journal
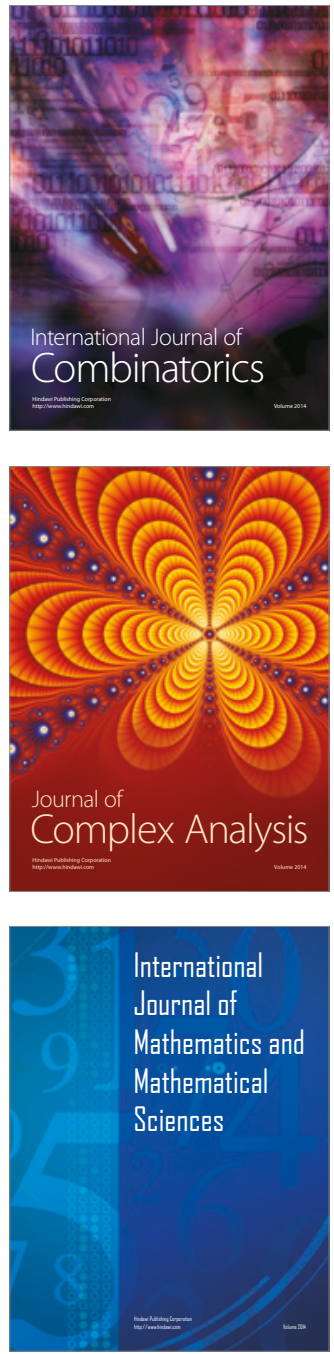
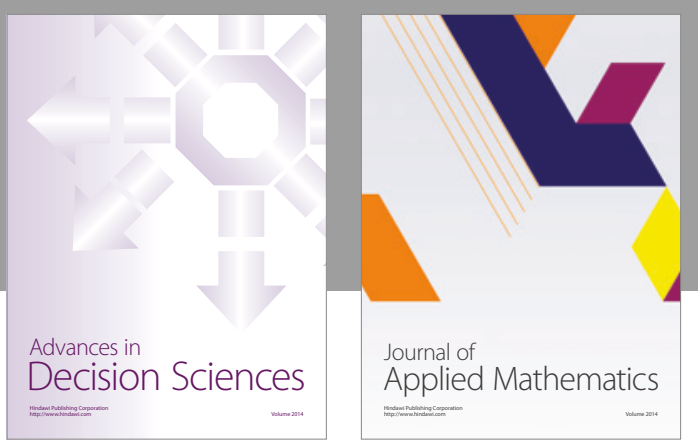

Algebra

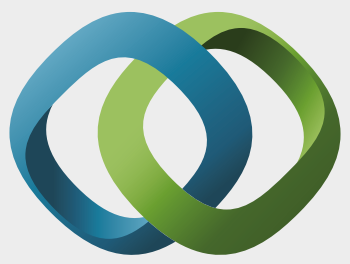

\section{Hindawi}

Submit your manuscripts at

https://www.hindawi.com
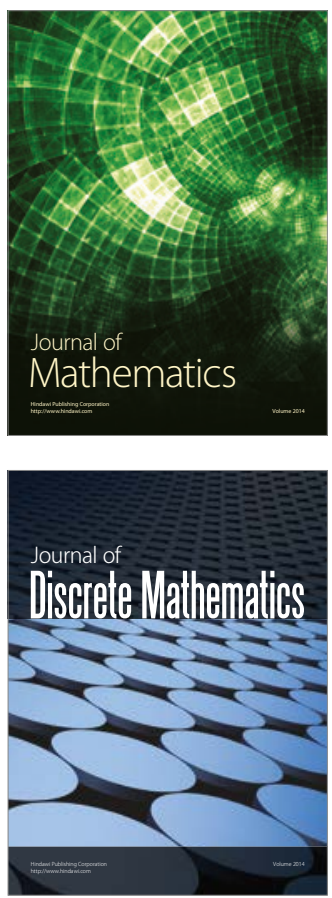

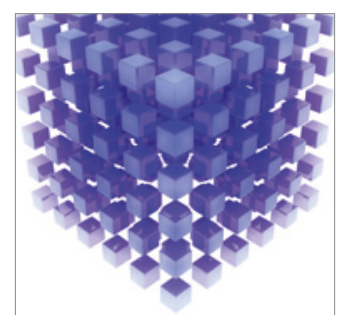

Mathematical Problems in Engineering
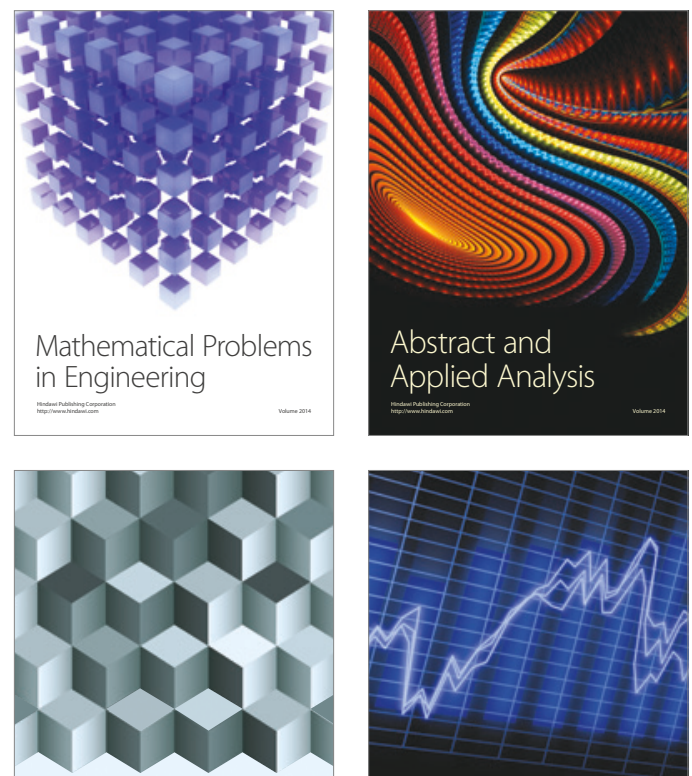

Journal of

Function Spaces

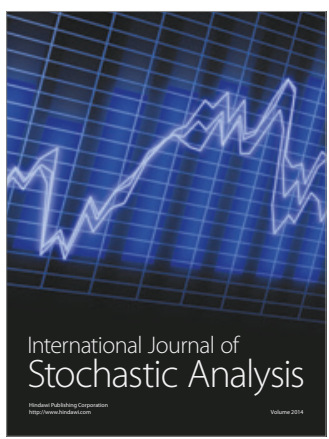

Probability and Statistics
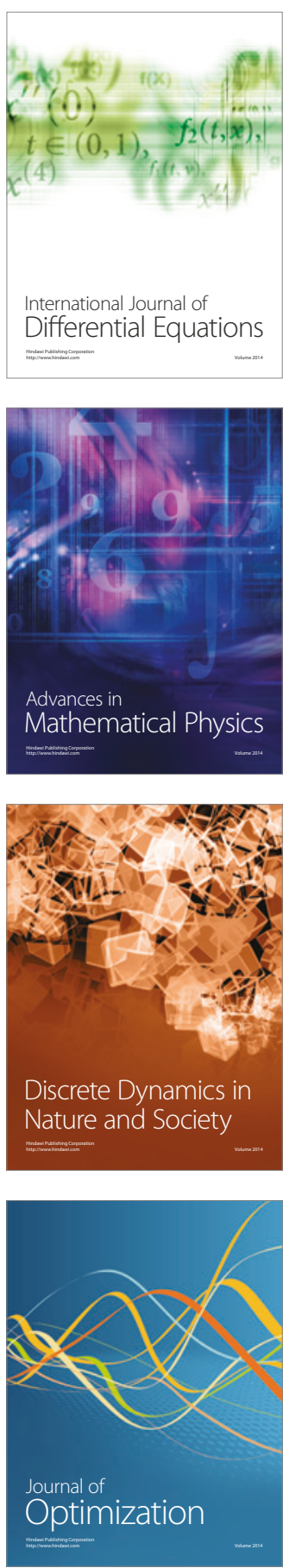